\title{
EX Lupi in quiescence ${ }^{\star}$
}

\author{
N. Sipos ${ }^{1}$, P. Ábrahám ${ }^{1}$, J. Acosta-Pulido ${ }^{2}$, A. Juhász ${ }^{3}$, Á. Kóspál ${ }^{4}$, M. Kun ${ }^{1}$, A. Moór ${ }^{1}$, and J. Setiawan ${ }^{3}$ \\ ${ }^{1}$ Konkoly Observatory of the Hungarian Academy of Sciences, PO Box 67, 1525 Budapest, Hungary \\ e-mail: niki@konkoly.hu \\ 2 Instituto de Astrofísica de Canarias, E-38200 La Laguna, Tenerife, Canary Islands, Spain \\ 3 Max-Planck-Institut für Astronomie, Königstuhl 17, 69117 Heidelberg, Germany \\ ${ }^{4}$ Leiden Observatory, Leiden University, PO Box 9513, 2300RA Leiden, The Netherlands
}

Received 9 January 2009 / Accepted 4 June 2009

ABSTRACT

\begin{abstract}
Aims. EX Lupi is the prototype of EXors, a subclass of low-mass pre-main sequence stars whose episodic eruptions are attributed to temporarily increased accretion. In quiescence the optical and near-infrared properties of EX Lup cannot be distinguished from those of normal T Tau stars. Here we investigate whether it is the circumstellar disk structure that makes EX Lup an atypical Class II object. During outburst the disk might undergo structural changes. Our characterization of the quiescent disk is intended to serve as a reference for studying the physical changes related to one of EX Lupi's strongest known eruptions in 2008 Jan-Sep.

Methods. We searched the literature for photometric and spectroscopic observations including ground-based, IRAS, ISO, and Spitzer data. After constructing the optical-infrared spectral energy distribution (SED), we compared it with the typical SEDs of other young stellar objects and modeled it using the Monte Carlo radiative transfer code $R A D M C$. We determined the mineralogical composition of the $10 \mu \mathrm{m}$ silicate emission feature and also gave a description of the optical and near-infrared spectra.

Results. The SED is similar to that of a typical T Tauri star in most aspects, though EX Lup emits higher flux above $7 \mu \mathrm{m}$. The quiescent phase data suggest low-level variability in the optical-mid-infrared domain. By integrating the optical and infrared fluxes, we derived a bolometric luminosity of $0.7 L_{\odot}$. The $10 \mu \mathrm{m}$ silicate profile could be fitted by a mixture consisting of amorphous silicates, but no crystalline silicates were found. A modestly flaring disk model with a total mass of $0.025 M_{\odot}$ and an outer radius of $150 \mathrm{AU}$ was able to reproduce the observed SED. The derived inner radius of $0.2 \mathrm{AU}$ is larger than the sublimation radius, and this inner gap sets EX Lup apart from typical T Tauri stars.
\end{abstract}

Key words. stars: formation - stars: circumstellar matter - stars: individual: EX Lup - infrared: stars

\section{Introduction}

In 2008 January the amateur astronomer A. Jones announced that EXLupi, the prototype of the EXor class of pre-main sequence eruptive variables, had brightened dramatically (Jones 2008). The quiescent phase brightness of EX Lup, an M0 V star in the Lupus 3 star-forming region, is $V \approx 13 \mathrm{mag}$. During its unpredictable flare-ups, its brightness may increase by 1-5 mag for a period of several months (Herbig 1977). In past decades EX Lup produced a number of eruptions, the last one in 2002 (Herbig 1977; Herbig et al. 2001; Herbig 2007). During the present outburst, which lasted until 2008 September (AAVSO International Database $^{1}$ ), EX Lup reached a peak brightness of 8 mag in 2008 January, brighter than ever before.

According to the current paradigm, eruptive phenomena in pre-main sequence stars (FU Ori and EX Lup-type outbursts) are caused by enhanced accretion onto the central star. Intense buildup of the stellar mass takes place during these phases of the star formation process (Hartmann \& Kenyon 1996). In this picture circumstellar matter must play a crucial role in the eruption mechanism. Surprisingly little is known about the circumstellar environment of the EXor prototype, EXLup. While its infrared excess emission detected by IRAS and ISO was attributed

^ Full Table 3 is only available in electronic form at the CDS via anonymous ftp to cdsarc.u-strasbg.fr (130.79.128.5) or via http://cdsweb.u-strasbg.fr/cgi-bin/qcat?]/A+A/507/881

1 www .aavso.org to a circumstellar disk (Gras-Velázquez \& Ray 2005), no detailed analysis or modeling of the disk structure has been performed so far. Such an analysis could contribute to clarifying the eruption mechanism and also answering the longstanding open question of what distinguishes EXors from normal T Tau stars. Herbig (2008) found no optical spectroscopic features that would uniquely define EXors, and he also concluded that their location in the $(J-H)$ vs. $\left(H-K_{\mathrm{s}}\right)$ color-color diagram coincides with the domain occupied by $\mathrm{T}$ Tau stars.

In this work we search the literature and construct a complete optical-infrared spectral energy distribution (SED) of EXLup that is representative of the quiescent phase. The SED is modeled using a radiative transfer code and will be compared with typical SEDs of T Tau stars, in order to reveal differences possibly defining the EXor class. During the recent extreme eruption, EX Lupi was observed with a wide range of instruments. Our results on the physical parameters of the system can be used as a reference for evaluating the changes related to this outburst.

\section{Optical / infrared data}

\subsection{Optical and near-infrared photometry from the literature}

We searched the literature and collected available optical and near-infrared photometric observations of EX Lup obtained during its quiescent phase. We defined quiescence periods as when the source was fainter than 12.5 mag according to the visual 
Table 1. Optical and infrared observations of EXLup collected from the literature, obtained in its quiescent periods.

\begin{tabular}{|c|c|c|c|}
\hline$\overline{\bar{\lambda} \lambda[\mu \mathrm{m}]}$ & Julian date & $\overline{\overline{\text { Magnitude }}}$ & $\overline{\text { Reference }}$ \\
\hline \multirow{8}{*}{$0.36(U)$} & 2443349.545 & 14.21 & 1 \\
\hline & 2443349.572 & 14.2 & 1 \\
\hline & 2443349.681 & 14.23 & 1 \\
\hline & 2443349.717 & 14.25 & 1 \\
\hline & 2443349.728 & 14.28 & 1 \\
\hline & 2443350.653 & 14.22 & 1 \\
\hline & 2443353.659 & 14.13 & 1 \\
\hline & 2443895.85 & 14.52 & 2 \\
\hline \multirow[t]{10}{*}{$0.44(B)$} & 2443349.545 & 14.33 & 1 \\
\hline & 2443349.572 & 14.36 & 1 \\
\hline & 2443349.681 & 14.24 & 1 \\
\hline & 2443349.717 & 14.36 & 1 \\
\hline & 2443349.728 & 14.33 & 1 \\
\hline & 2443350.653 & 14.20 & 1 \\
\hline & 2443353.659 & 14.07 & 1 \\
\hline & 2443894.85 & 14.52 & 2 \\
\hline & 2443895.85 & 14.42 & 2 \\
\hline & 2448896 & 14.21 & 3 \\
\hline \multirow{10}{*}{$0.55(V)$} & 2443349.545 & 13.13 & 1 \\
\hline & 2443349.572 & 13.19 & 1 \\
\hline & 2443349.681 & 13.27 & 1 \\
\hline & 2443349.717 & 13.27 & 1 \\
\hline & 2443349.728 & 13.21 & 1 \\
\hline & 2443350.653 & 13.14 & 1 \\
\hline & 2443353.659 & 13.22 & 1 \\
\hline & 2443894.85 & 13.20 & 2 \\
\hline & 2443895.85 & 13.22 & 2 \\
\hline & 2448896 & 13.03 & 3 \\
\hline $0.64(R)$ & 2448896 & 12.15 & 3 \\
\hline $0.79(I)$ & 2451295.81 & 11.109 & 4 \\
\hline $1.25(\mathrm{~J})$ & 2441474 & 9.76 & 5 \\
\hline $1.25(J)$ & 2445075 & 9.92 & 6 \\
\hline $1.235(J)$ & 2451314.781 & 9.728 & 7 \\
\hline $1.221(J)$ & 2451295.81 & 9.92 & 4 \\
\hline $1.65(H)$ & 2441474 & 9.04 & 5 \\
\hline $1.65(H)$ & 2445075 & 9.11 & 6 \\
\hline $1.662(H)$ & 2451314.781 & 8.958 & 7 \\
\hline $2.2(K)$ & 2441474 & 8.82 & 5 \\
\hline $2.2(K)$ & 2445075 & 8.78 & 6 \\
\hline $2.159\left(K_{\mathrm{s}}\right)$ & 2451314.781 & 8.496 & 7 \\
\hline $2.144\left(K_{\mathrm{s}}\right)$ & 2451295.81 & 8.82 & 4 \\
\hline $3.4(L)$ & 2441474 & $\geq 8.7$ & 5 \\
\hline $3.4(L)$ & 2445075 & 8.05 & 6 \\
\hline $4.8(M)$ & 2445075 & 7.54 & 6 \\
\hline
\end{tabular}

References: (1) Bastian \& Mundt (1979); (2) Mundt \& Bastian (1980); (3) Herbig et al. (1992); (4) The DENIS database (DENIS Consortium 2005); (5) Glass \& Penston (1974); (6) Hughes et al. (1994); (7) 2MASS All-Sky Catalog of Point Sources (Cutri et al. 2003).

estimates and $V$-band magnitudes in the AAVSO International Database. A visual inspection of the DSS and the 2MASS images revealed no nebulosity surrounding the star, so we concluded that measurements taken with different apertures can be safely compared. The query results are presented in Table 1 and plotted in Fig. 1.

\subsection{ESO 2.2/FEROS optical spectroscopy}

Three spectra of EXLup in quiescent phase were taken in 2007 July with FEROS at the 2.2 m MPG/ESO telescope in ESO La Silla, Chile. FEROS has a spectral resolution of 48000 and a wavelength coverage from 360 to $920 \mathrm{~nm}$ (Kaufer et al. 1999). The data was reduced using the online FEROS Data Reduction
System implemented on the telescope, which produced 39 one dimensional spectra of echelle orders and a merged spectrum of the entire wavelength range.

With a cross-correlation method, we computed the projected rotational velocity and radial velocity of EXLup in quiescence from the FEROS spectra. We cross-correlated the spectra with a numerical template mask (Baranne et al. 1996). The mean radial velocity is $-0.948 \pm 0.1 \mathrm{~km} \mathrm{~s}^{-1}$. From the $B-V$ color of EX Lup and by using a projected rotational velocity calibration for FEROS (Setiawan et al. 2004), we derived a $v \sin i=$ $4.4 \pm 2 \mathrm{~km} \mathrm{~s}^{-1}$. Sections of the spectrum showing the most prominent emission lines are plotted in Fig. 2.

\subsection{NTT/SOFI near-infrared spectroscopy}

We found near-IR spectra in the ESO Data Archive, obtained on 2001 May 4 using SOFI at the ESO $3.5 \mathrm{~m}$ NTT telescope, under the program 67.C-0221(A) (PI:D. Folha). Two spectra were taken using the blue and red grisms $(R \sim 1000)$, which cover the ranges $0.95-1.64$ and $1.53-2.52 \mu \mathrm{m}$, respectively. In both cases, the total exposure time was $500 \mathrm{~s}$, divided into 4 exposures following an ABBA nodding pattern. The data were reduced using our dedicated routines developed in the IRAF environment. The data reduction included sky-subtraction, wavelength-calibration, and frame combination. Spectra of the G3V star HIP 78466 were used to correct for telluric absorption. A modified version of the Xtellcor software (Vacca et al. 2003) was used in this step. The spectrum is presented in Fig. 3.

\subsection{InfraRed Astronomical Satellite (IRAS)}

EXLup is included in the IRAS Point Source Catalog with detections at 12,25 and $60 \mu \mathrm{m}$, and with an upper limit at $100 \mu \mathrm{m}$. The measurements took place during a quiescent phase of EXLup. In order to refine these data and also try to obtain a $100 \mu \mathrm{m}$ flux density value, we analyzed the IRAS raw scans again using the IRAS Scan Processing and Integration tool (SCANPI) available at IPAC ${ }^{2}$. Depending on the wavelength, 6-8 scans crossed the object. The scans were re-sampled and averaged, and a peak was located within $30^{\prime \prime}$ of the position of EX Lup. Then a point-source template was fitted and fluxes calculated. The flux errors were dominated by the uncertainty in estimating the background level. The fluxes were color-corrected in an iterative way by convolving the source SED and the IRAS filter profiles. The color-corrected fluxes and flux uncertainties can be seen in Table 2.

\subsection{Infrared Space Observatory (ISO)}

EX Lup was observed on 5 dates with ISOPHOT, the photometer onboard ISO (Lemke et al. 1996). Four measurements belong to a small monitoring program that adopted similar instrumental setups at each epoch (PI: T. Prusti). For such datasets, an algorithm aimed to achieve high relative photometry accuracy was developed in our group (Juhász et al. 2007). The raw data were corrected for instrumental effects following the scheme described in Juhász et al. (2007). Absolute flux calibration was performed by using the onboard calibration source (FCS) for the $3.6-12 \mu \mathrm{m}$ filters and by assuming a default responsivity for the $20-25 \mu \mathrm{m}$ range. At different single epochs 60, 100, and $200 \mu \mathrm{m}$ high-resolution maps were performed using the Astronomical

\footnotetext{
2 http://scanpiops.ipac.caltech.edu:9000/applications/ Scanpi/
} 


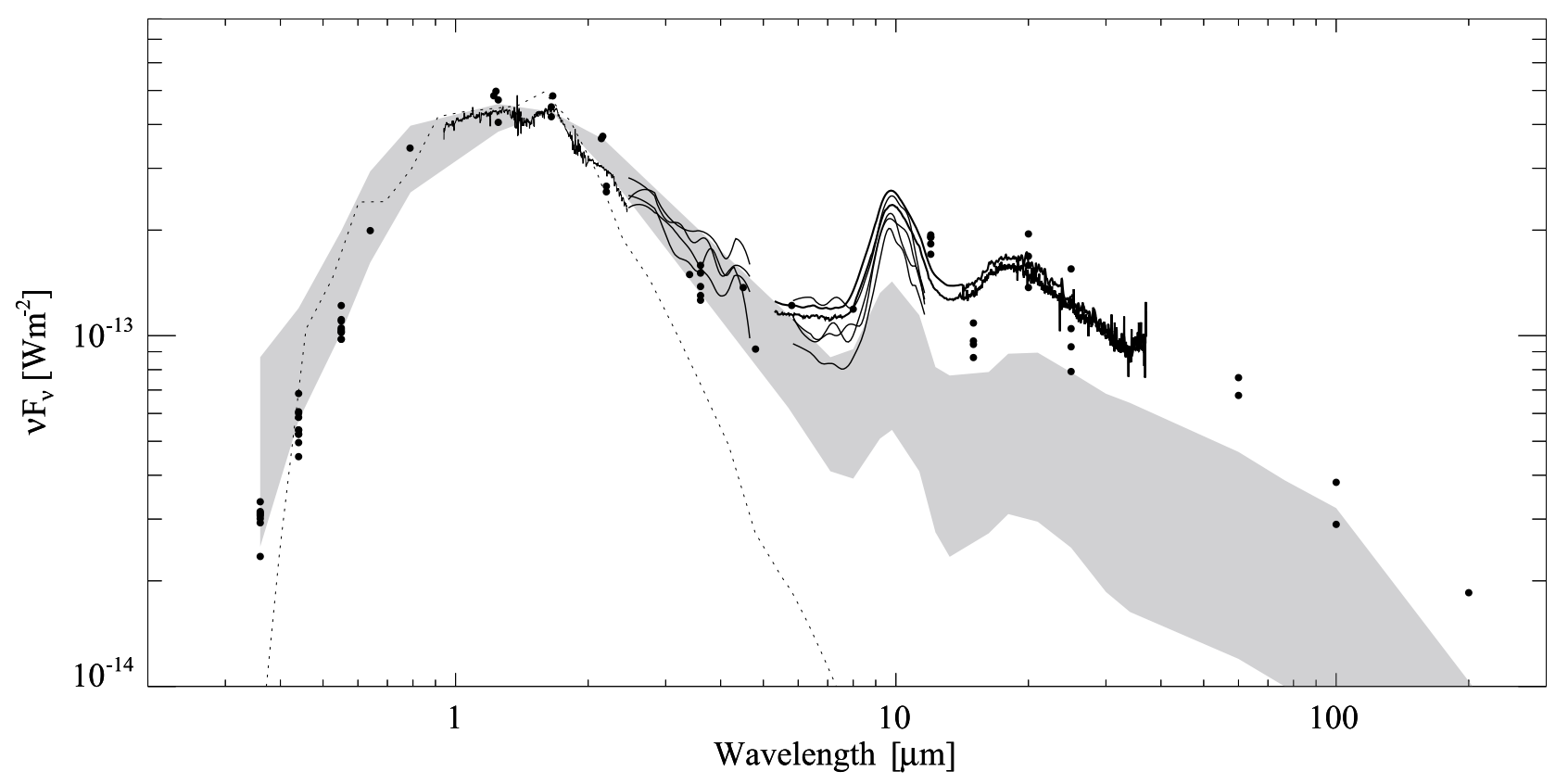

Fig. 1. Spectral energy distribution of EX Lup, showing all data points from Tables 1 and 2 and the smoothed spectra obtained with NTT/SOFI, ISOPHOT-S, and Spitzer/IRS. The gray stripe marks the median SED of T Tauri objects from the Taurus-Auriga star-forming region. The 5-36 $\mu \mathrm{m}$ section of the median SED was constructed by Furlan et al. (2006) based on Spitzer IRS data of 55 Class II objects with spectral types between K5 and M2, while the remaining part of our median SED was taken from D'Alessio et al. (1999) who computed their SED from 39 T Tauri stars. The dotted line overplots the stellar photosphere used in the models explained in Sect. 4.3.
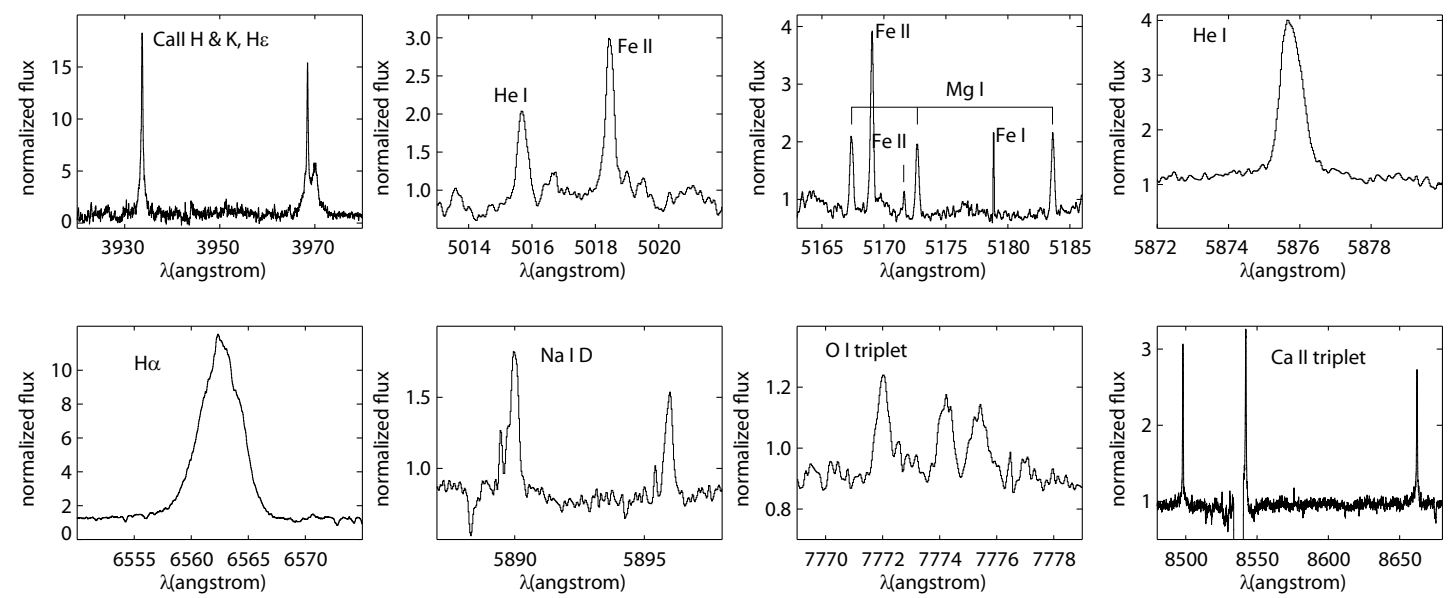

Fig. 2. Details of the optical spectrum of EX Lupi showing significant or typical features. The interval shown in the second panel of the upper row is the same as Herbig's (2007) Fig. 3. It is apparent that the broad component of the Fe II $\lambda 5018$ line was absent in 2007. The third panel shows a typical region with narrow metallic lines. The O I $\lambda 7773$ triplet, shown in the third panel of the lower row, was observed in absorption in several T Tauri stars (Hamann \& Persson 1992).

Observing Template P32. These data were processed using a dedicated P32 Tool (Tuffs \& Gabriel 2003) and the calibration used the related FCS measurements. The flux values were colorcorrected by convolving the SED of EX Lup with the ISOPHOT filter profiles. The results are listed in Table 2, where the quoted formal uncertainties correspond to a conservative estimate of $15 \%$; however, the measurement error above $25 \mu \mathrm{m}$ is probably higher than this value. The fifth photometric dataset was published by Gras-Velázquez \& Ray (2005), but these data will not be used in our analysis due to their presumably higher uncertainties.

At the four epochs of the monitoring program low-resolution spectrophotometry was also performed with the ISOPHOT-S sub-instrument in the $2.5-11.6 \mu \mathrm{m}$ wavelength range. These observations were reduced following the processing scheme described by Kóspál et al. (2009a, in prep.), which corrected for the slight off-center positioning of the source, subtracted the separate background spectra, and adopted realistic error bars. The processed and calibrated spectra are shown in Fig. 1.

\subsection{Spitzer Space Telescope}

IRAC. EXLup was observed with IRAC at 3.6, 4.5, 5.8, and $8.0 \mu \mathrm{m}$ on 2005 March 29 (PID: 3716, PI: G. Stringfellow), in the subarray mode. At each of the nine dither positions, 64 images were obtained with an exposure time of $0.1 \mathrm{~s}$. The BCD (S14.0) image cubes of the 64 frames were combined into twodimensional images using the "irac-subcube-collapse" IDL routine provided by the Spitzer Science Center. Aperture photometry was performed on the nine final images at each wavelength 
Table 2. Color-corrected fluxes of EX Lupi* .

\begin{tabular}{lcccccc}
\hline \hline$\lambda[\mu \mathrm{m}]$ & $\begin{array}{c}1983 \\
\text { IRAS }\end{array}$ & $\begin{array}{c}\text { 1997 Feb. 5 } \\
\text { ISOPHOT }\end{array}$ & $\begin{array}{c}\text { 1997 Mar. 18 } \\
\text { ISOPHOT }\end{array}$ & $\begin{array}{c}\text { 1997 Aug. 24 } \\
\text { ISOPHOT }\end{array}$ & $\begin{array}{c}\text { 1997 Sept. 19 } \\
\text { ISOPHOT }\end{array}$ & $\begin{array}{c}\text { 2005 Mar. 29 } \\
\text { Spitzer/IRAC }\end{array}$ \\
\hline 3.6 & & $0.17 \pm 0.03$ & $0.18 \pm 0.03$ & $0.15 \pm 0.02$ & $0.16 \pm 0.03$ & $0.190 \pm 0.004$ \\
4.5 & & & & & & $0.206 \pm 0.004$ \\
5.8 & & & & & & $0.236 \pm 0.005$ \\
8.0 & & & & & $0.317 \pm 0.007$ \\
12 & $0.76 \pm 0.1$ & $0.73 \pm 0.11$ & $0.78 \pm 0.12$ & $0.76 \pm 0.12$ & $0.68 \pm 0.11$ & \\
15 & & $0.54 \pm 0.08$ & $0.43 \pm 0.07$ & $0.47 \pm 0.07$ & $0.48 \pm 0.07$ & \\
20 & & $1.06 \pm 0.15$ & $1.30 \pm 0.18$ & $0.91 \pm 0.13$ & $1.12 \pm 0.16$ & \\
25 & $1.04 \pm 0.1$ & $0.78 \pm 0.12$ & $0.87 \pm 0.14$ & $0.66 \pm 0.11$ & $1.29 \pm 0.21$ & \\
60 & $1.35 \pm 0.4$ & $1.52 \pm 0.32$ & & & & \\
100 & $0.97 \pm 0.5$ & & $1.27 \pm 0.17$ & & & \\
200 & & & & $1.23 \pm 0.35$ & & \\
\hline
\end{tabular}

${ }^{*}$ For the ISOPHOT data we adopted a conservative formal uncertainty of $15 \%$.

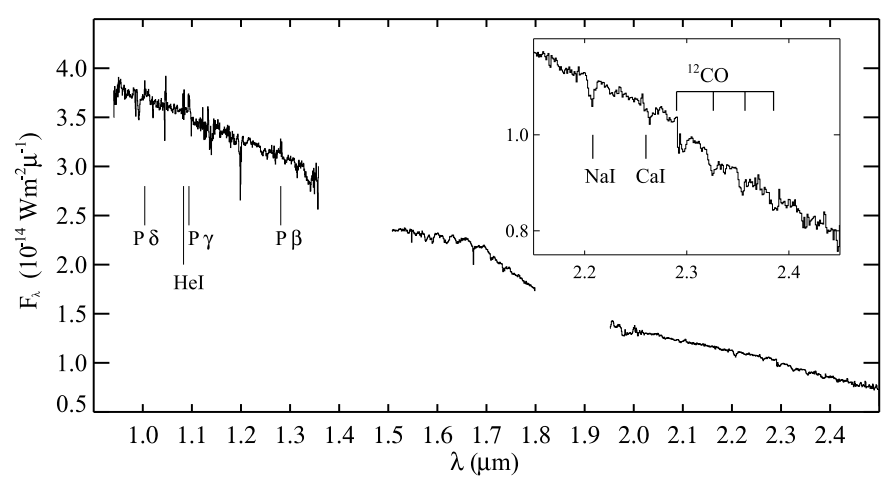

Fig. 3. Near-infrared spectrum of EX Lupi, observed on 2001 May 4. The region around the ${ }^{12} \mathrm{CO}$ bandhead absorption is enlarged in the inset.

using a modified version of the IDLPHOT routines. The aperture radius was set to 3 pixels $\left(3.66^{\prime \prime}\right)$, the sky background was computed in an annulus with an inner radius of 10 pixels and a width of 10 pixels. (Sigma-clipping was used to reject outlying pixel values.) The aperture correction used the quoted values from the IRAC Data Handbook (V3.0, hereafter IDH). Following the outline of the IDH (see Chap. 5) in the $3.6 \mu \mathrm{m}$ band a pixel-phase correction was also applied to the measured flux densities. The flux density values measured in the nine dither positions (at each band) were averaged to get the final photometry. To calculate the final uncertainty, we quadratically added the measurement errors (obtained from the nine individual flux density values in each band) and an absolute calibration error of $2 \%$ (IDH).

IRS. EX Lup was observed with the Infrared Spectrograph (IRS, Houck et al. 2004) of the Spitzer Space Telescope on 2004 Aug. 30 (PID: 172, PI: N. Evans) and on 2005 March 18 (PID:3716, PI: G. Stringfellow). On the first date, the target was measured using Short Low $(5.2-14.5 \mu \mathrm{m})$, Long Low $(14.0-38 \mu \mathrm{m})$, Short High $(9.9-19.5 \mu \mathrm{m})$, and Long High $(18.7-37.2 \mu \mathrm{m})$ modules. The integration time was $14 \mathrm{~s}$ for the low-resolution modules, while $30 \mathrm{~s}$ and $60 \mathrm{~s}$ were used for the Short High and Long High modules, respectively. At the second epoch only the Short Low, Short High, and Long High modules were used and a PCRS peak-up was executed prior to the spectroscopic observation to acquire the target in the spectrograph slit. The integration times were $14 \mathrm{~s}$ for the Short Low module with 4 observing cycles for redundancy, while $120 \mathrm{~s}$ and $60 \mathrm{~s}$ were used for the Short High and Long High modules, respectively, both with 2 observing cycles.

The spectra are based on the droopres and rsc products processed through the S 15.3.0 version of the Spitzer data pipeline for the low- and high-resolution data, respectively. For the low-resolution spectra, the background was subtracted using associated pairs of imaged spectra from the two nodded positions, also eliminating stray light contamination and anomalous dark currents. For the high-resolution spectra, the background was removed by fitting a local continuum underneath the source profile. Pixels flagged by the data pipeline as being "bad" were replaced with a value interpolated from an 8 pixel perimeter surrounding the flagged pixel. The spectra were extracted using a 6.0 pixel and 5.0 fixed-width aperture in the spatial dimension for the Short Low and the Long Low modules, respectively, while in the case of the high-resolution modules the spectra were extracted by fitting the source profile with the known PSF in the spectral images. The low-level fringing at wavelengths $>20 \mu \mathrm{m}$ was removed using the irsfringe package (Lahuis \& Boogert 2003). The spectra were calibrated with a spectral response function derived from IRS spectra and MARCS stellar models for a suite of calibrators provided by the Spitzer Science Center. To remove any effect of pointing offsets, we matched orders based on the point spread function of the IRS instrument, correcting for possible flux losses. The spectra obtained at the two different dates agree within an uncertainty of $\leq 11 \%$ and are shown in Fig. 1.

\section{Results}

\subsection{Variability in the quiescent phase}

The observations of EX Lup are sporadic, so no complete SED could be constructed for any given epoch. We decided to merge all quiescent data regardless of their dates. In Fig. 1 we plotted all fluxes listed in Tables 1 and 2. At most wavelengths, the scatter of data points is on the order of $25 \%$, though at optical and a few mid-infrared wavelengths, the difference between the lowest and highest values can be considerably larger. The ISOPHOT-S and Spitzer spectra shown in Fig. 1 also exhibit differences at similar levels. Although part of this scatter is related to instrumental and calibration effects, the individual error bars are typically much smaller than the scatter of the data points, strongly suggesting the existence of an intrinsic variability. However, in the case of the 60 and $100 \mu \mathrm{m}$ data points, the uncertainty of the measurements is much higher than at shorter wavelengths, so here the difference between the fluxes is within the error bars. 
Table 3. Observed wavelength, $E W$, and $F W H M$ of the most prominent emission lines in the optical spectrum of EX Lupi in quiescence*.

\begin{tabular}{lcrr}
\hline \hline Line & $\begin{array}{c}\lambda \\
(\AA)\end{array}$ & $\begin{array}{r}E W \\
(\AA)\end{array}$ & $\begin{array}{r}F W H M \\
\left(\mathrm{~km} \mathrm{~s}^{-1}\right)\end{array}$ \\
\hline $\mathrm{Ca}$ II H & 3933.66 & -13.09 & 45.8 \\
$\mathrm{Ca}$ II K & 3968.46 & -5.41 & 22.7 \\
$\mathrm{H} \delta$ & 4101.70 & -14.29 & 155.1 \\
$\mathrm{H} \gamma$ & 4340.42 & -13.02 & 137.0 \\
$\mathrm{H} \beta$ & 4861.25 & -18.74 & 144.0 \\
$\mathrm{He} \mathrm{I}(11)$ & 5875.75 & -1.96 & 37.6 \\
$\mathrm{Na} \mathrm{I}(1)$ & 5889.96 & -0.52 & 22.9 \\
$\mathrm{Na}$ I (1) & 5895.94 & -0.34 & 18.3 \\
$\mathrm{H} \alpha$ & 6562.56 & -35.95 & 191.0 \\
$\mathrm{HeI}(46)$ & 6678.24 & -0.67 & 23.8 \\
$\mathrm{He}$ I (10) & 7065.31 & -0.42 & 29.3 \\
$\mathrm{Ca}$ II (2) & 8498.01 & -1.51 & 23.0 \\
$\mathrm{Ca}$ II (2) & 8542.08 & -2.26 & 40.5 \\
$\mathrm{Ca}$ II (2) & 8662.17 & -1.49 & 27.0 \\
\hline
\end{tabular}

* The full list of the emission lines identified in the spectrum are found in the electronic version of this table at the CDS.

We consider the $25 \%$ peak-to-peak variation as an upper limit for the variability of EX Lup in quiescence. According to Fig. 1, the variability might be somewhat greater at optical wavelengths, but the low number of data points prevents us from claiming a wavelength dependence.

\subsection{The optical-infrared SED}

The measured SED of EX Lup is presented in Fig. 1. The optical part is clearly dominated by the stellar photosphere. The optical and near-infrared colors, however differ slightly from the standard colors of an M0 V star. This fact has already been mentioned by Gras-Velázquez \& Ray (2005), who could not derive a positive extinction value from the $E_{B-V}$ and $E_{R-I}$ colors. Similarly, Herbig et al. (2001) claim it is unknown as well. An infrared excess above the photosphere is detectable longwards of the $K$-band. The $3-8 \mu \mathrm{m}$ range is smooth and devoid of any broad spectral features, indicating that EX Lup is neither deeply embedded to exhibit ices nor hot enough to excite PAHs. A strong silicate emission appears at $10 \mu \mathrm{m}$, and a corresponding - though broader and shallower - silicate band can be seen around $20 \mu \mathrm{m}$. At longer wavelengths, the continuum emission decreases following a power law with a spectral index of about $-4 / 3$. The lack of any sub-mm or mm measurement means that the SED cannot be followed longwards of $200 \mu \mathrm{m}$.

\subsection{Spectroscopy}

\subsubsection{The optical spectrum}

The high-resolution optical spectrum of EX Lupi, obtained on 2007 July 30, shows several emission lines in addition to the photospheric absorption features characteristic of the young latetype star. The emission lines are mostly symmetric, without noticeable P Cygni or inverse P Cygni absorption. The most prominent emission lines are the Balmer lines of the hydrogen, the $\mathrm{H}$ and $\mathrm{K}$ lines and the infrared triplet of the ionized calcium, and the helium lines at 5875 and $6678 \AA$. The observed wavelengths and equivalent widths in $\AA$ and the full widths at half maximum in $\mathrm{km} \mathrm{s}^{-1}$, determined by Gaussian fitting, are listed in Table 3. In addition to these prominent features, more than 200 weak, narrow metallic lines could be identified using Moore's (1945) multiplet tables. The widths of the lines are around $10-20 \mathrm{~km} \mathrm{~s}^{-1}$, indicating that they originate in the active chromosphere of the star (Hamann \& Persson 1992). All the emission lines identified in the spectrum are listed in the electronic version of Table 3 at the Centre de Données astronomiques de Strasbourg (CDS). Figure 2 shows sections of the spectrum.

The shape of the $\mathrm{H} \alpha$ line is nearly symmetric. Its equivalent width, $W(\mathrm{H} \alpha)=-35.9 \AA$, is significantly larger than the upper limit of the chromospheric $\mathrm{H} \alpha$ emission of M0 type stars ( 6 A , Barrado y Navascués \& Martín 2003), indicating active accretion during the quiescent phase. The velocity width of the $\mathrm{H} \alpha$ line $10 \%$ above the continuum level is $362 \mathrm{~km} \mathrm{~s}^{-1}$, larger than the lower limit of $270 \mathrm{~km} \mathrm{~s}^{-1}$, set by White \& Basri (2003) for accreting T Tauri stars. The empirical relationship between the $10 \%$ width of the $\mathrm{H} \alpha$ line and the accretion rate $\dot{M}_{\text {acc }}$, found by Natta et al. (2004), allowed us to derive $\dot{M}_{\text {acc }}=4.2_{-2.3}^{+8.1} \times$ $10^{-10} M_{\odot} / \mathrm{yr}$. Comparison of the $\mathrm{H} \alpha$ line with the one published by Reipurth, Pedrosa \& Lago (1996) shows that in 2007 the $\mathrm{H} \alpha$ emission line of EX Lupi was more symmetric narrower and had somewhat larger equivalent width than in 1994 March, a few days after an outburst.

\subsubsection{Near-infrared spectrum}

The flux-calibrated infrared spectrum, observed on 2001 May 4, is displayed in Fig. 3. The shape of the spectrum is similar to that of an unreddened M-type star without near-infrared excess (Greene \& Lada 1996). In the ZJ band the Paschen $\beta, \gamma, \delta$, and the He I $\lambda 1.083 \mu \mathrm{m}$ lines are seen in emission. Absorption features of the Na I at $2.21 \mu \mathrm{m}, \mathrm{Ca}$ I at $2.26 \mu \mathrm{m}$, and the rovibrational $\Delta v=+2$ transitions of $\mathrm{CO}$ at $2.3-2.4 \mu \mathrm{m}$ can be identified in the $K$-band part of the spectrum. The $\mathrm{Br} \gamma$ line of the hydrogen is barely visible in emission, but its equivalent width cannot be measured. The following absorption features are also identified in our spectrum: at $1.199 \mu \mathrm{m}$ a very deep feature corresponding to S I; at $1.577 \mu \mathrm{m}$ a feature associated to $\mathrm{Mg} \mathrm{I}$, and at $1.673 \mu \mathrm{m}$ another feature associated with Al I.

The $\operatorname{Pa} \beta$ emission line is a useful accretion tracer, because its luminosity correlates well with the accretion luminosity (e.g. Muzerolle et al. 1998; Dahm 2008). We used the relationship established by Muzerolle et al. (1998) to derive the accretion luminosity of EX Lup during quiescence from the measured flux $F(\operatorname{Pa} \beta)=1.7 \times 10^{-17} \mathrm{~W} \mathrm{~m}^{-2}$. The resulting $L_{\mathrm{acc}}=3.7 \times 10^{-3} L_{\odot}$ allowed us to determine the mass accretion rate $\dot{M}_{\text {acc }}$, taking the mass and the radius of the star from Table 6 . The accretion was assumed to proceed through a gaseous disk; for its inner radius we adopted $R_{\text {in }} \approx 5 R_{*}$ (Gullbring et al. 1998). The result is $\dot{M}_{\text {acc }} \approx 4.0 \times 10^{-10} M_{\odot} /$ yr, in agreement with $\dot{M}_{\text {acc }}$ obtained from the velocity width of the $\mathrm{H} \alpha$ line. A flux calibration uncertainty of $20 \%$ and the uncertainty of the empirical relationship sets the lower and upper limits at $1.3 \times 10^{-10} M_{\odot} / \mathrm{yr}$ and $1.1 \times 10^{-9} M_{\odot} / \mathrm{yr}$, respectively.

\section{Discussion}

\subsection{Comparison with young stellar objects}

Infrared SEDs of eruptive young stars (EXors and/or FUors) were examined by Green et al. (2006), Quanz et al. (2007) and Kóspál et al. (2009b, in prep.). Some of these stars (eg. UZ Tau, VY Tau, DR Tau, FU Ori, Bran 76) resemble EXLupi. Their SEDs decrease towards longer wavelengths and show a silicate feature in emission. The SEDs of the other group of FU Ori-type 
or EXor-like variables (e.g. V1057 Cyg, V1647 Ori, PV Cep, OO Ser) exhibit flat or increasing SEDs in the $20-100 \mu \mathrm{m}$ wavelength range (Ábrahám et al. 2004; Kóspál et al. 2007), which is clearly different from the shape of EX Lupi's spectral energy distribution. Green et al. (2006) and Quanz et al. (2007) suggest that the diversity in the shape of the SEDs is related to the evolution of the system. Younger objects, still embedded in a large envelope exhibit flat SEDs, while more evolved objects, emitting T Tauri-like SEDs have already lost their envelopes and only have circumstellar disks. According to this categorization we conclude that EX Lupi is relatively evolved among eruptive stars, and expect that its circumstellar environment only consists of a disk without an envelope.

To compare EX Lup with other - not eruptive - young stars, in Fig. 1 we overplotted a gray stripe marking the median SED of $\mathrm{T}$ Tauri objects from the Taurus-Auriga star-forming region (D'Alessio et al. 1999; Furlan et al. 2006). The SED of EX Lupi follows the Taurus median at shorter wavelengths. Longwards of approximately $7 \mu \mathrm{m}$, however, its absolute level becomes higher than the median by a factor of $\sim 2.5$. Nevertheless, even in this longer wavelength range its shape still resembles the Taurus slope.

Another comparison of EXLupi with other pre-main sequence stars can be based on the mid-infrared spectrum. Furlan et al. (2006) present a large sample of Spitzer IRS spectra of young stars and group them into several categories by measuring the slope of the SEDs and the strength of the 10 and $20 \mu \mathrm{m}$ silicate features. A visual classification places EX Lup in their scheme in group A or in group B, defined by a relatively strong silicate feature and a flat or somewhat decreasing SED over $20 \mu \mathrm{m}$. Furlan et al. (2006) suggest that only limited dust-growth and settling has taken place in these groups. Nonetheless, we find that EXLup is not a typical member of these groups, since shortwards of $8 \mu \mathrm{m}$ the steep decrease of excess emission characteristic of their objects is missing in the case of EXLupi. These qualitative conclusions can be verified by calculating color indices for EX Lup. The strength of its silicate feature $\left(F_{10}-F_{\text {cont }}\right) / F_{\text {cont }}=0.58$ is an intermediate value between group A and group B objects. However, the $n_{6-25}$ spectral index is typically negative for all objects in the sequence while positive (0.03) in the case of EX Lupi, making it an "outlier" in the scheme. Also, the correlation between the $n_{6-13}$ and $n_{13-25}$ indices found by Furlan et al. (2006) does not seem to hold for EX Lup. The colors of IRAS 04385+2550, a star exhibiting IRS spectrum very similar to that of EXLup, were interpreted by Furlan et al. (2006) as indicating for the opening of an inner gap in the disk. This might also give an explanation in case of EX Lup for the lower $6 \mu \mathrm{m}$ flux compared to that at longer wavelengths. Other sources from their sample that resemble EX Lup are UY Aur, CZ Tau, and HP Tau. It is interesting to note that all four objects are binary systems with different separations.

Finally we calculated the $T_{\text {bol }}$ bolometric temperature and $L_{\text {bol }}$ bolometric luminosity of EX Lup following the method of Chen et al. (1995). They studied the Taurus and Ophiuchus starforming regions, and analyzed the distribution of young stars of different evolutionary stages in the $L_{\text {bol }}$ vs. $T_{\text {bol }}$ diagram. In a later paper (Chen et al. 1997), they repeated the study for the objects of the Lupus cloud as well, including EX Lup. They found $T_{\text {bol }}=2365 \mathrm{~K}$ and $L_{\text {bol }}=0.7 L_{\odot}$ based on IRAS data. Using our newly constructed SED, we recalculated these two values. Assuming $A_{V}=0 \mathrm{mag}$, our result is $T_{\text {bol }}=1982 \mathrm{~K}$ and $L_{\text {bol }}=0.73 \mathrm{~L}_{\odot}$. According to these parameters, EX Lup seems to be a typical classical T Tauri star. The location of EX Lup on the
Table 4. Overview of dust species used in fitting the 5-17 $\mu \mathrm{m}$ spectrum*.

\begin{tabular}{lllll}
\hline \hline Species & State & $\begin{array}{l}\text { Chemical } \\
\text { formula }\end{array}$ & Shape & Ref. \\
\hline $\begin{array}{l}\text { Amorphous silicate } \\
\text { (olivine stoichiometry) }\end{array}$ & $\mathrm{A}$ & $\mathrm{MgFeSiO}_{4}$ & $\begin{array}{l}\text { Homogeneous } \\
\text { sphere }\end{array}$ \\
$\begin{array}{l}\text { Amorphous silicate } \\
\text { (pyroxene stoichiometry) }\end{array}$ & $\mathrm{A}$ & $\mathrm{MgFeSi}_{2} \mathrm{O}_{6}$ & $\begin{array}{l}\text { Homogeneous } \\
\text { sphere }\end{array}$ \\
$\begin{array}{l}\text { Forsterite } \\
\begin{array}{l}\text { Clino Enstatite } \\
\text { Silica }\end{array}\end{array}$ & $\mathrm{C}$ & $\mathrm{Mg}_{2} \mathrm{SiO}_{4}$ & $\begin{array}{l}\text { Hollow sphere } \\
\text { (2) }\end{array}$ \\
\hline
\end{tabular}

* References: (1) Dorschner et al. (1995); (2) Servoin \& Piriou (1973); (3) Jäger et al. (1998); (4) Henning \& Mutschke (1997).

$L_{\text {bol }}$ vs. $T_{\text {bol }}$ diagram implies that it is a Class II object, and its age is $3.8_{-2.7}^{+9.2} \times 10^{6} \mathrm{yr}$.

\subsection{Mineralogy}

Based on the previous results that EX Lup is similar to classical T Tauri stars, in the following we assume that its circumstellar matter is distributed in a disk. To derive the dust composition in the surface layer of the disk, we fitted the Spitzer IRS spectrum using the Two-Layer Temperature Distribution (TLTD) method (Juhász et al. 2009). This method assumes multicomponent continuum (star, inner rim, disk midplane) and a distribution of temperatures to fit the source function. The silicate emission is expected to arise from the disk atmosphere. The observed spectrum is computed as

$F_{v}=F_{v, \text { cont }}+\sum_{i=1}^{N} \sum_{j=1}^{M} D_{i, j} K_{i, j} \int_{T_{\text {atm,max }}}^{T_{\text {atm,min }}} \frac{2 \pi}{d^{2}} B_{v}(T) T^{\frac{2-\text { qatm }}{\text { qatm }}} \mathrm{d} T$,

where

$$
\begin{aligned}
F_{v, \text { cont }}= & \frac{\pi R_{\star}}{d^{2}} B_{v}\left(T_{\star}\right) \\
& +D 1 \int_{T_{\text {rim,max }}}^{T_{\text {rim,min }}} \frac{2 \pi}{d^{2}} B_{v}\left(T_{\text {rim }}\right) T_{\text {rim }}{ }^{\frac{2-q r i m}{\text { qrim }}} \mathrm{d} T \\
& +D 2 \int_{T_{\text {mid,max }}}^{T_{\text {mid,min }}} \frac{2 \pi}{d^{2}} B_{v}\left(T_{\text {mid }}\right) T_{\text {mid }} \frac{2-\text { qmid }}{\text { qmid }} \mathrm{d} T .
\end{aligned}
$$

Here, $d$ is the distance, $B_{v}(T)$ the Planck-function and $\kappa_{i, j}$ the mass absorption coefficient of dust species $i$ and grain size $j$. The subscripts "rim", "atm" and "mid" denote the quantities of the inner rim, the disk atmosphere, and the disk mid-plane, respectively. In Table 4 we list the used dust species. For each component we specify its lattice structure, chemical composition, shape and reference to the laboratory measurements of the optical constants. For the homogeneous spheres we used Mie theory to calculate the mass absorption/scattering coefficients. For the inhomogeneous spheres, we used the distribution of hollow spheres (Min et al. 2005), to simulate grain shape deviating from perfect symmetry.

In Fig. 4 we present the fit to the $5-17 \mu$ m region of the Spitzer IRS spectrum. The derived dust mass fractions are given in Table 5. The spectral decomposition shows that the main contributors to the optically thin $10 \mu \mathrm{m}$ silicate emission complex are the amorphous silicates $(98.4 \pm 1.0 \%$ in terms of mass $)$. The mass fraction of crystalline silicates is below $2 \%$, which agrees with the value for the diffuse ISM (Kemper et al. 2005). It is interesting to note that the mass-weighted average grain size of the 


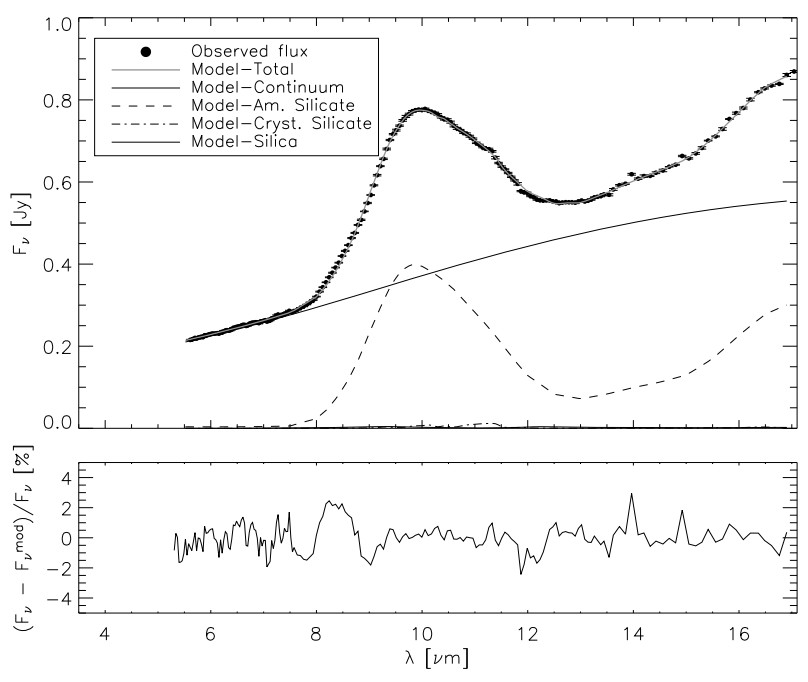

Fig. 4. Fit of the observed spectrum. The spectral decomposition shows that the mid-infrared spectrum of EX Lup can be reproduced by a mixture of amorphous silicates with olivine and pyroxene stoichiometry. The contribution of crystalline silicates derived from the spectral decomposition is below $2 \%$.

amorphous silicates $(0.57 \mu \mathrm{m})$ in our fit is somewhat larger than typically found in the ISM. At wavelengths longer than $17 \mu \mathrm{m}$, the IRS spectrum of EX Lup only shows the $18 \mu \mathrm{m}$ feature of the amorphous silicates, and no crystalline feature can be seen.

The fitted values for $T_{\text {rim,max }}, T_{\text {atm,max }}$, and $T_{\text {mid,max }}$ are $1202 \pm$ $4 \mathrm{~K}, 1032 \pm 90 \mathrm{~K}$, and $846 \pm 4 \mathrm{~K}$, respectively. The fitted values for $q_{\text {rim }}, q_{\text {atm }}$, and $q_{\text {mid }}$ are $-2.02 \pm 0.01,-0.44 \pm 0.02$, and $-0.42 \pm 01$, respectively. Although the $\chi^{2}$ of the fit is 14.5 , which is far higher than unity, as expected for a good fit, the average deviation from the observed spectrum is about $1 \%$. The reason for the high $\chi^{2}$ can be found in the relatively high signal-to-noise ratio of the Spitzer IRS spectrum ( $>300$ in the fitted wavelength range) and in the deficiencies of the applied dust model (optical constants and grain-shape model). The latter is probably responsible for the differences between the 2D RT model and the observed IRS spectrum between 14 and $21 \mu \mathrm{m}$.

Given that EX Lup is a young eruptive star, one would expect to observe an increased value for crystallinity in the midinfrared features, compared to "normal" T Tauri stars. Since the only requirement of the crystallization is the high temperature, the enhanced irradiation luminosity and viscous heating during the outbursts should lead to rapid crystallization in the disk. In accordance with our results, Quanz et al. (2007) report the lack of crystalline emission and the presence of emission from larger grains $(a>0.1 \mu \mathrm{m})$ in the mid-infrared spectra of FU Orionis objects. They hypothesize that the reason for the lack of crystals can be twofold. One can be the replenishment of the dust content of the disk atmosphere (where the mid-infrared features originate) by pristine dust from an infalling envelope of the FU Ori object. Another possible explanation is strong vertical mixing in the disk that transports the crystals into deeper layers of the disk where they cannot be detected any longer by mid-infrared spectroscopy. Out of these two speculative scenarios, the latter is favorable for EX Lup, since to our knowledge, there is no infalling envelope around the source.

\subsection{Modeling}

In this section we perform a detailed modeling of the EX Lup system, to derive the geometry of its circumstellar environment.
Table 5. Fitted dust composition to the Spitzer IRS spectrum, with mass fractions larger than $0.1 \%$.

\begin{tabular}{lccc}
\hline \hline Dust species & \multicolumn{3}{c}{ Mass fraction [\%] } \\
& $0.1 \mu \mathrm{m}$ & $1.5 \mu \mathrm{m}$ & $6.0 \mu \mathrm{m}$ \\
\hline $\begin{array}{l}\text { Am. Silicate } \\
\text { (Olivine type) }\end{array}$ & $65.6 \pm 0.5$ & - & - \\
$\begin{array}{l}\text { Am. Silicate } \\
\text { (Pyroxene type) }\end{array}$ & - & $32.80 \pm 0.5$ & - \\
Forsterite & $0.7 \pm 0.08$ & - & - \\
Enstatite & - & $0.5 \pm 0.3$ & $0.4 \pm 0.4$ \\
Silica & - & - & - \\
\hline
\end{tabular}

We fit the data points presented in Fig. 1, except the midinfrared domain where, due to the intrinsic variability and the different quality of the data, we considered only the Spitzer measurements: the IRAC and IRS observations form a quasisimultaneous high quality data set covering the $3.6-38 \mu \mathrm{m}$ wavelength range. Moreover, these measurements are close in time to the recent 2008 eruption and thus document the preoutburst conditions of the system.

For the stellar parameters (radius $R_{*}$, effective temperature $T_{*}$, and mass $M_{*}$ ) we adopted the values used by Gras-Velázquez \& Ray (2005). These parameters were fixed during the modeling. (Table 6 lists all input parameters marking differently the fixed and the optimized ones.) For the distance of the star we adopted $155 \mathrm{pc}$, the mean distance of the Lupus Complex as measured by the Hipparcos (Lombardi et al. 2008). The unknown location of EX Lup within the complex introduces an additional uncertainty of about $30 \mathrm{pc}$. For the value of extinction we assumed $A_{V}=0$ mag (Sect. 3.2).

We used the Monte Carlo radiative transfer code $R A D M C$ (Dullemond \& Dominik 2004) combined with RAYTRACE ${ }^{3}$. The circumstellar environment is supposed to be axially symmetric, so we used a two-dimensional geometry in polar coordinates $(r, \theta)$. As mentioned in Sects. 2.1 and 4.1 we had no indication of an envelope around EXLupi, thus our system only consists of a central star and a dusty disk. For the structure of the disk we assume the density profile as

$\rho_{\text {disk }}(r, z)=\frac{\Sigma(r)}{h(r) \sqrt{2 \pi}} \exp \left\{-\frac{1}{2}\left[\frac{z}{h(r)}\right]^{2}\right\}$,

where $r$ and $z$ are the radial and vertical coordinates, respectively, and $\Sigma(r)=\Sigma_{\text {disk }}\left(\frac{r}{r_{\text {disk }}}\right)^{-p}$ is the surface density. The "disk" subscript denotes values of the given parameter at the outer radius. The scale height $h(r)$ increases with radius as

$\frac{h(r)}{r}=\frac{h_{\mathrm{disk}}}{r_{\mathrm{disk}}}\left(\frac{r}{r_{\mathrm{disk}}}\right)^{\alpha_{\mathrm{fl}}}$,

where $\alpha_{\mathrm{fl}}$ is the flaring index. A schematic picture of the geometry is presented in Fig. 5.

The temperature distribution is determined by the heating sources: the central star, for which we used a Kurucz model atmosphere (Castelli \& Kurucz 2003), and the heated dust grains emitting blackbody radiation. We used a passive disk and did not consider accretion, due to the low accretion rate in quiescence (Sect. 3.3). The accretion luminosity is $<1 \%$ of the stellar luminosity, thus its contribution is negligible compared to direct

\footnotetext{
3 For details see: http://www.mpia.de/homes/dullemon/ radtrans/radmc/
} 


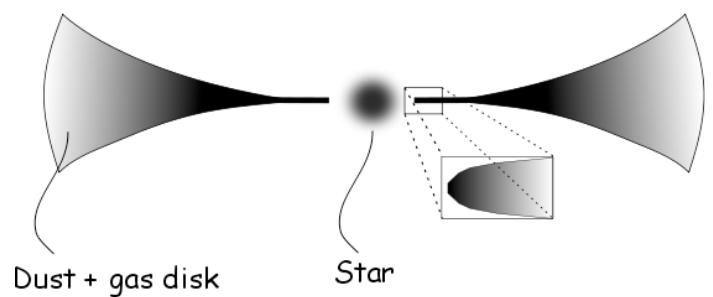

Fig. 5. Schematic picture of the geometrical structure of the model. The figure is not to scale.

irradiation coming from the central star. After the calculation of the temperature distribution, the SED of the system is produced at an inclination angle $\vartheta$ with the ray-tracer.

We used the dust composition derived from the fitting of the Spitzer IRS spectrum (Sect.4.2), but excluded dust species whose contribution to the total mass is $\leq 1 \%$. This way, the dust model used in $R A D M C$ contained only amorphous silicates of olivine and of pyroxene types with a mass ratio of $2: 1$. The size of the dust grains were $0.1 \mu \mathrm{m}$ and $1.5 \mu \mathrm{m}$ for olivine and pyroxene stoichiometry, respectively. Besides these, we added $20 \%$ amorphous carbon with a grain size of $0.1 \mu \mathrm{m}$. The mass absorption coefficients of amorphous carbon were calculated using Mie-theory from the optical constants of Preibisch et al. (1993).

We could reasonably fit most of the measured data points by adopting the geometry described above. In the best model of this type we had to move the inner radius of the dusty disk out to $0.5 \mathrm{AU}$, significantly exceeding the dust sublimation radius (at $T=1500 \mathrm{~K}$ ) of less than 0.1 AU. However, in the $3-8 \mu \mathrm{m}$ wavelength range this model underestimated the measured points (Fig. 6, dotted line). Using smaller values for the inner radius, we could improve the fit of the $3-5 \mu \mathrm{m}$ range, but then it was not possible to reproduce the measured fluxes at longer wavelengths (Fig. 6, dashed line). Reducing the inner radius below 0.2 AU the model fluxes even in the near-infrared wavelength range became too high. To solve this problem, we introduced a rounded inner rim to the disk (see inset in Fig. 5); thus instead of having a sharp inner edge with a high wall, we decreased the disk height to $\frac{h_{\text {disk }}}{r_{\text {disk }}}=0.05$ at the beginning of the disk and at the same time moved the inner radius inward. Behind 0.6 AU we left the structure of the disk unchanged. With this modification we could obtain a fit that is in good agreement with all infrared observations. Nevertheless, the inner radius of $0.2 \mathrm{AU}$ used in the best model is still beyond the sublimation radius.

Our best-fit model is presented in Fig. 6 with a solid line, and the corresponding parameters listed in Table 6. In the literature we could not find any constraints for the inclination of the system, and its value is not defined well by the model either. The disk is definitely seen closer to face-on then edge-on, and we obtained our best fit using a value of $20^{\circ}$, though fits for inclinations between $0^{\circ}$ (face on) and $40^{\circ}$ are very similar. The outer radius of the circumstellar disk is typical of young systems, while the disk is massive among T Tauri disks. For the value of the flaring index $\alpha_{\mathrm{fl}}=0.09$ gave the best result, which means that the disk only flares very modestly. The scale height is $h=12 \mathrm{AU}$ at the outer radius of the disk, which is slightly higher than the typical value for $\mathrm{T}$ Tauri disks. The exponent of the radial surface density profile is $p=-1.0$.

\section{Summary and conclusions}

We characterized the quiescent disk of EX Lupi and investigated whether it is the structure of the circumstellar environment that

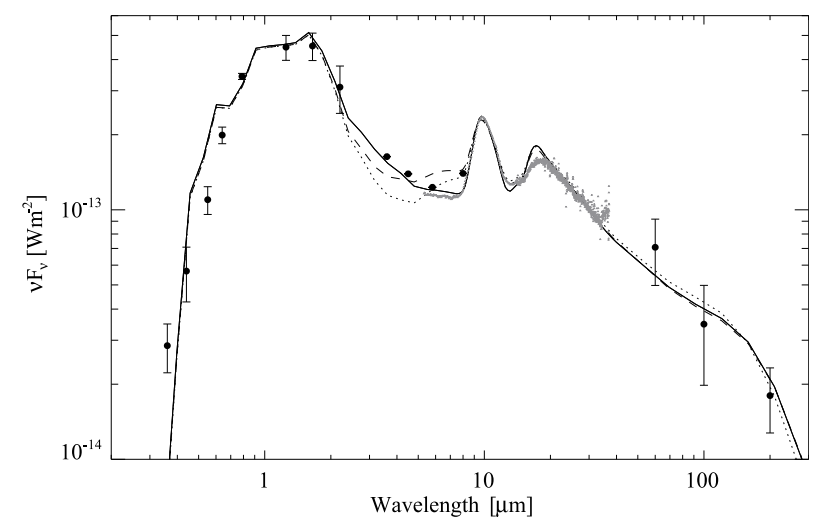

Fig. 6. Spectral energy distribution of EX Lup. The solid line shows our best fit model with the rounded inner wall, while the dashed and dotted lines correspond to the best models using a sharp inner edge. The plotted error bars mark the range of observed fluxes at different epochs, also taking the individual measurement uncertainties into account (filled dots correspond to the middle of the range). Error bars on the IRAC points are smaller then the size of the symbols.

Table 6. Parameters used in the best-fit model, where those in italics were adopted from the literature and kept fixed during the modeling.

\begin{tabular}{lc}
\hline \hline Parameters & Fitted value \\
\hline System Parameters & \\
Distance $(d)$ & $155 \mathrm{pc}$ \\
Inclination $(\vartheta)$ & $20^{\circ}$ \\
Stellar Parameters & $3800 \mathrm{~K}$ \\
Temperature $\left(T_{\text {star }}\right)$ & $0.6 M_{\odot}$ \\
Mass $\left(M_{\text {star }}\right)$ & $1.6 \mathrm{R}_{\odot}$ \\
Radius $\left(R_{\text {star }}\right)$ & $0 \mathrm{mag}$ \\
Visual extinction $\left(A_{V}\right)$ & \\
Circumstellar Disk Parameters & $0.2 \mathrm{AU}$ \\
Inner radius of dusty disk $\left(r_{\text {in,disk }}\right)$ & $150 \mathrm{AU}$ \\
Outer radius of dusty disk $\left(r_{\text {disk }}\right)$ & 0.12 \\
Scale height $\left(\frac{h_{\text {disk }}}{r_{\text {disk }}}\right)$ & 0.09 \\
Flaring index $\left(\alpha_{\text {fl }}\right)$ & -1.0 \\
Exponent of radial density profile $(p)$ & $0.025 M_{\odot}$ \\
Total mass $(M)$ & \\
\hline
\end{tabular}

makes it an atypical, eruptive young stellar object. Our main findings are the following:

- During quiescent phase there is an indication of an intrinsic variability of less than $25 \%$ in the optical-mid-infrared wavelength regime.

- Our new spectra are consistent with the classification of EXLup as an $\mathrm{M}$ type star. Based on the $\mathrm{H} \alpha$ and $\mathrm{Pa} \beta$ spectral lines, we derived a very low quiescent accretion rate of $\sim 4 \times 10^{-10} M_{\odot} / \mathrm{yr}$.

- In general the shape of the SED is similar to those of typical T Tau stars, but above $7 \mu \mathrm{m}$ EX Lup is brighter than the Taurus median by a factor of $\sim 2.5$. The relative flux contribution from shorter and longer wavelengths is a parameter that may distinguish EX Lup from the majority of classical T Tau stars.

- The $10 \mu \mathrm{m}$ silicate feature of EX Lup can be reproduced well by amorphous silicates with olivine and pyroxene stoichiometry, but no crystalline silicates were found.

- A modestly flaring disk model with a total mass of $0.025 M_{\odot}$ and with inner and outer radii of 0.2 and $150 \mathrm{AU}$, respectively, is able to reproduce the observed SED. The radius of the inner hole is larger than the dust sublimation radius. 
The existence of this dust-free inner hole points to a clearing mechanism, for which several explanations can be invoked:

1. Eisner et al. (2007) claim that low-mass young stars with low accretion luminosities tend to have inner disk radii larger than the sublimation radius, probably due to magnetic field effects. Using the relationship in Eisner et al. (2007) and taking $0.2 \mathrm{AU}$ as the magnetospheric inner radius and an accretion rate of $4 \times 10^{-10} M_{\odot} / y r$ EX Lupi should have a magnetic field strength of $2.3 \mathrm{kG}$, which is typical of T Tauri stars (Johns-Krull 2007).

2. Binarity might also be responsible for clearing up regions of the disk. The case of EXLup binarity has been studied by several authors. Ghez et al. (1997) used high angular resolution techniques to find wide components, and detected no companion of EXLup between 150-1800 AU separation. Bailey (1998) detected no companion between 1-10 AU. Melo (2003) claims that EXLup is not a spectroscopic binary, and similarly Herbig (2007) concludes the same based on spectroscopic data measured by the Keck telescope. Guenther et al. (2007) performed an eight-year long radial velocity monitoring program, but they could not detect binarity in the case of EX Lupi either though they only had 3 spectra. Nevertheless, we note that, if the inclination of EX Lupi is indeed close to a face-on geometry, it makes it difficult to detect a binarity.

3. Inner gaps are characteristic of disks in the transitional phase between the Class II and Class III stages. These transitional disks, unlike EX Lup, exhibit no excess over the photosphere in the near-infrared wavelength range. Furlan et al. (2006) suggest that IRAS $04385+2550$, an object whose SED is similar to that of EXLup, could be in a state preceding the transition. This explanation would mean that EXLup might also be in a pre-transitional state.

4. Alternatively, with such a low accretion rate, photoevaporation by EUV radiation may also contribute to the clearing of this innermost region (Gorti \& Hollenbach 2009).

5. It cannot be excluded that the large inner hole is a pheanomenon connected with the eruptive behavior, although details of this connection are unclear yet.

Our detailed modeling of the quiescent disk structure of EX Lup (Sect. 4.2) may be a good basis for studying the physical changes related to the 2008 eruption. Assuming that the outburst stems from temporarily increased accretion, one could scale up the accretion rate in our quiescent model and check whether this new model would reproduce the outburst SED. If this strategy fails to provide a good enough fit to the outburst data, it may be a hint of geometrical restructuring of the circumstellar environment during the outburst.

One of our main goals in the present work was to identify atypical features in the circumstellar structure of EX Lup, which may explain its eruptive nature. The inner-disk hole revealed by our modeling is an unexpected result in this sense and comparison of EX Lup with other EXors at infrared wavelengths would be important. It may answer the question of whether an inner gap in the dusty disk is characteristic of the EXor phenomenon connecting the hole to the eruption mechanism, and we could learn to what extent EX Lup is a good representative of eruptive stars.

Acknowledgements. The work was supported by the grants OTKA T 49082 and OTKA K 62304 of the Hungarian Scientific Research Fund. The authors are grateful to C.P. Dullemond for kindly providing RAYTRACE and also to Jeroen Bouwman for providing his routines for the Spitzer IRS data reduction. The research of Á.K. is supported by the Nederlands Organization for
Scientific Research. They also acknowledge for observational data from the AAVSO International Database contributed by observers worldwide.

\section{References}

Ábrahám, P., Kóspál, Á., Csizmadia, S., et al. 2004, A\&A, 419, L39 Bailey, J. 1998, MNRAS, 301, 161

Baranne, A., Queloz, D., Mayor, M., et al. 1996, A\&AS, 119, 373

Barrado y Navascués, D., \& Martín, E. L. 2003, AJ, 126, 2997

Bastian, U., \& Mundt, R. 1979, A\&AS, 36, 57

Castelli, F., \& Kurucz, R. L. 2003, Modelling of Stellar Atmospheres, 210, 20P

Chen, H., Myers, P. C., Ladd, E. F., \& Wood, D. O. S. 1995, ApJ, 445, 377

Chen, H., Grenfell, T. G., Myers, P. C., \& Hughes, J. D. 1997, ApJ, 478, 295

Cutri, R. M., et al. 2003, The IRSA 2MASS All-Sky Point Source Catalog, NASA/IPAC Infrared Science Archive

D’Alessio, P., Calvet, N., Hartmann, L., Lizano, S., \& Cantó, J. 1999, ApJ, 527, 893

Dahm, S. E. 2008, AJ, 136, 521

The Denis Consortium 2005, VizieR Online Data Catalog, 1, 2002

Dorschner, J., Begemann, B., Henning, Th., Jäger, C., \& Mutschke, H. 1995, A\&A, 300, 503

Dullemond, C. P., \& Dominik, C. 2004, A\&A, 417, 159

Dullemond, C. P., \& Turolla, R. 2000, A\&A, 360, 1187

Eisner, J. A., Hillenbrand, L. A., White, R. J., et al. 2007, ApJ, 669, 1072

Furlan, E., Hartmann, L., Calvet, N., et al. 2006, ApJS, 165, 568

Ghez, A. M., McCarthy, D. W., Patience, J. L., \& Beck, T. L. 1997, ApJ, 481, 378

Glass, I. S., \& Penston, M. V. 1974, MNRAS, 167, 237

Gorti, U., \& Hollenbach, D. 2009, ApJ, 690, 1539

Gras-Velázquez, À., \& Ray, T. P. 2005, A\&A, 443, 541

Green, J. D., Hartmann, L., Calvet, N., et al. 2006, ApJ, 648, 1099

Greene, T. P., \& Lada, C. J. 1996, AJ, 112, 2184

Guenther, E. W., Esposito, M., Mundt, R., et al. 2007, A\&A, 467, 1147

Gullbring, E., Hartmann, L., Briceno, C., \& Calvet, N. 1998, ApJ, 492, 323

Hamann, F., \& Persson, S. E. 1992, ApJS, 82, 285

Hartigan, P., \& Kenyon, S. J. 2003, ApJ, 583, 334

Hartmann, L., \& Kenyon, S. J. 1996, ARA\&A, 34, 207

Henning, Th., \& Mutschke, H. 1997, A\&A, 327, 743

Herbig, G. H. 1977, ApJ, 217, 693

Herbig, G. H. 2007, AJ, 133, 2679

Herbig, G. H. 2008, AJ, 135, 637

Herbig, G. H., Suntzeff, N., \& Blanco, B. 1992, Information Bulletin on Variable Stars, 3755,1

Herbig, G. H., Aspin, C., Gilmore, A. C., Imhoff, C. L., \& Jones, A. F. 2001, PASP, 113, 1547

Hughes, J., Hartigan, P., Krautter, J., \& Kelemen, J. 1994, AJ, 108, 1071

Jäger, C., Molster, F. J., Dorschner, J., et al. 1998, A\&A, 339, 904

Johns-Krull, C. M. 2007, ApJ, 664, 975

Jones, A. F. A. L. 2008, Central Bureau Electronic Telegrams, 1217, 1

Juhász, A., Prusti, T., Ábrahám, P., \& Dullemond, C. P. 2007, MNRAS, 374, 1242

Juhász, A., Henning, Th., Bouwman, J., et al. 2009, ApJ, accepted

Kaufer, A., Stahl, O., Tubbesing, S., et al. 1999, The Messenger, 95, 8

Kemper, F., Vriend, W., \& Tielens, A. 2005, ApJ, 633, 534

Kóspál, Á., Ábrahám, P., Prusti, T., et al. 2007, A\&A, 470, 211

Kóspál, Á., Németh, P., Ábrahám, P., et al. 2008, Information Bulletin on Variable Stars, 5819, 1

Kóspál, Á., Ábrahám, P., Kun, M., et al. 2009a, in prep.

Kóspál, Á., et al. 2009b, in prep.

Lahuis, F., \& Boogert, A. 2003, SFChem 2002: Chemistry as a Diagnostic of Star Formation, 335

Lemke, D., Klaas, U., Abolins, J., et al. 1996, A\&A, 315, L64

Lombardi, M., Lada, C. J., \& Alves, J. 2008, A\&A, 480, 785

Melo, C. H. F. 2003, A\&A, 410, 269

Min, M., Hovenier, J., \& de Koter, A. 2005, A\&A, 432, 909

Moore, C. E. 1945, Contrib. Princeton Univ. Obs., No. 20

Mundt, R., \& Bastian, U. 1980, A\&AS, 39, 245

Muzerolle, J., Hartmann, L., \& Calvet, N. 1998, AJ, 116, 2965

Natta, A., Testi, L., Muzerolle, J., et al. 2004, A\&A, 424, 603

Preibisch, T., Ossenkopf, V., Yorke, H., \& Henning, Th. 1993, A\&A, 279, 577

Quanz, S. P., Henning, Th., Bouwman, J., et al. 2007, ApJ, 668, 359

Reipurth, B., Pedrosa, A., \& Lago, M. T. V. T. 1996, A\&AS, 120, 229

Servoin, J. L., \& Piriou, B. 1973, Phys. Stat. Sol., 55, 677

Setiawan, J., Pasquini, L., da Silva, L., et al. 2004, A\&A, 421, 241

Tuffs, R. J., \& Gabriel, C. 2003, A\&A, 410, 1075

Vacca, W. D., Cushing, M. C., \& Rayner, J. T. 2003, PASP, 115, 389

White, R. J., \& Basri, G. 2003, ApJ, 582, 1109

White, R. J., \& Ghez, A. M. 2001, ApJ, 556, 265 